Bull. Austral. Math. Soc.

$65 \mathrm{~F} 10,65 \mathrm{~F} 15,65 \mathrm{~B} 99$

VoL. 39 (1989) [477-478]

\title{
Extrapolation Methods and Iterative Computation of \\ Derivatives of Eigensystems
}

ROGER C.E. TAN

Many problems in engineering and physical sciences require numerical computation of partial derivatives of eigenvalues and eigenvectors of parameter dependent matrices. Following a suggestion of Rudisill and Chu [4], this thesis explores the possible use of iterative methods for this problem. As originally proposed, Rudisill and Chu's method was not competitive with direct methods, and, apart from some analysis and extensions of Andrew [1, 2] has previously been neglected in the literature. This thesis shows that the competitive position of the iterative method can be improved dramatically by the use of certain extrapolation techniques. As well as making the iterative approach more competitive for this problem, our results also give more information about the extrapolation techniques used. This should be useful for many other applications.

Chapter I introduces the problem and describes various applications arising in engineering and physical sciences.

Chapter II surveys previously known methods. It also includes new numerical results for several methods and new theoretical results for an important direct method.

Chapter III describes the application of the scalar and vector $\varepsilon$-algorithms, which have not been previously applied to this problem. It is shown that, in the absence of roundoff, both algorithms yield the exact solution in a finite number of steps. The performance of both algorithms in the presence of roundoff is investigated. It is also shown that application of the vector $\varepsilon$-algorithm to this problem, for single eigenvalue, yields approximate values of all the other eigenvalues as a useful and almost free bonus.

Chapter IV describes the first successful implementation and application of the topological $\varepsilon$-algorithm of Brezinski [3]. This is a landmark development as, before this successful implementation, the potential usefulness of the topological $\varepsilon$-algorithm has been underestimated in the literature. Four versions of the topological $\varepsilon$-algorithm are given luere, two of which involve bilinear forms $[3]$ and the other two inner products $[8,10]$. Their numerical performances in the presence of roundoff are also compared. This chapter also corrects an important mistake of Smith, Ford and Sidi [5] who concluded that the topological $\varepsilon$-algorithm is not an effective numerical algorithm. When

Received 6 October 1988. Thesis submitted to La Trobe Univeristy, March 1988. Degree approved September 1988. Supervisor Dr. A. Andrew

Copyright Clearance Centre, Inc. Serial-fee code: 0004-9729/89 \$A2.00+0.00. 
this mistake is corrected, the topological $\varepsilon$-algorithm is shown to work well on all the examples considered in [5].

Chapter V describes several other extrapolation techniques. One of them is an extension of an extrapolation method proposed by Andrew [2].

In chapter VI, a simultaneous iteration scheme of Andrew [1] is analysed and extended and developed into an effective algorithm for numerical computation of partial derivatives of several eigenvalues and eigenvectors. The extrapolation methods described in the previous chapters when used with this simultaneous iteration technique are shown to be more stable than when used in conjuction with the simple iteration of Rudisill and Chu.

Finally chapter VII makes some suggestions for further research.

\section{REFERENCES}

[1] A.L. Andrew, 'Convergence of an iterative method for derivatives of eigensystems', J. Comput. Phys, 26 (1978), 107-112.

[2] A.L. Andrew, 'Iterative computation of derivatives of eigenvalues and eigenvectors', J. Inst. Math. Appl. 24 (1979), 209-218.

[3] C. Brezinski, 'Généralizations de la transformation de Shanks, de la table de Padé, et de l'e-algorithme', Calcolo 12 (1975), 317-360.

[4] C.S. Rudisill and X.Y. Chu, 'Numerical methods for evaluating the derivatives of eigenvalues and eigenvectors', AIAA J, 13 (1975), 834-837.

[5] D.A. Smith, W.F. Ford and A. Sidi, 'Extrapolation methods for vector sequences', SIAM Rev. 28 (1987), 199-233.

[6] R.C.E. Tan, 'Accelerating the convergence of an iterative method for derivatives of eigensystems', J. Comput. Phys. 67 (1986), 230-235.

[7] R.C.E. Tan, 'An extrapolation method for colnputing derivatives of eigensystems', Internat. J. Comput. Math. 22 (1987), 63-73.

[8] R.C.E. Tan, 'Computing derivatives of eigensystems by the topological e-algorithm', App. Numer. Math 3 (1987), 539-550.

[9] R.C.E. Tan, 'Computing derivatives of eigensystems by the vector e-algorithm', IMA J. Numer. Anal. 7 (1987), 485-494.

[10] R.C.E. Tan, 'Implementation of the topological E-algorithm', SIAM J. Sci. Statist. Comput. $\theta$ (1988), 839-848.

[11] R.C.E. Tan and A.L. Andrew, 'Computing derivatives of eigenvalues and eigenvectors by simulteneous iteration', IMA J. Numer. Anal. (1989) (to appear).

[12] R.C.E. Tan, 'Some acceleration methods for iterative computation of derivatives of eigenvalues and eigenvectors', Internat. J. Numer. Methods Engrg (1989) (to appear).

Department of Mathematics

National University of Singapore

10 Kent Ridge Crescent

Singapore 0511 